\title{
Innovation in Deep Space Habitat Interior Design: Lessons Learned from Small Space Design in Terrestrial Architecture
}

\author{
Matthew A. Simon \\ NASA Langley Research Center, Hampton, VA, 23681, USA \\ Larry Toups \\ NASA Johnson Space Center, Houston, TX, 77058 USA
}

\begin{abstract}
Increased public awareness of carbon footprints, crowding in urban areas, and rising housing costs have spawned a 'small house movement' in the housing industry. Members of this movement desire small, yet highly functional residences which are both affordable and sensitive to consumer comfort standards. In order to create comfortable, minimum-volume interiors, recent advances have been made in furniture design and approaches to interior layout that improve both space utilization and encourage multi-functional design for small homes, apartments, naval, and recreational vehicles. Design efforts in this evolving niche of terrestrial architecture can provide useful insights leading to innovation and efficiency in the design of space habitats for future human space exploration missions.

This paper highlights many of the cross-cutting architectural solutions used in small space design which are applicable to the spacecraft interior design problem. Specific solutions discussed include reconfigurable, multi-purpose spaces; collapsible or transformable furniture; multi-purpose accommodations; efficient, space saving appliances; stowable and mobile workstations; and the miniaturization of electronics and computing hardware. For each of these design features, descriptions of how they save interior volume or mitigate other small space issues such as confinement stress or crowding are discussed. Finally, recommendations are provided to provide guidance for future designs and identify potential collaborations with the small spaces design community.
\end{abstract}

\section{Nomenclature}

$\begin{aligned} \frac{\text { Acronyms }}{H I D H} & =\text { Human Integration Design Handbook } \\ \text { ISS } & =\text { International Space Station } \\ \text { LED } & =\text { Light Emitting Diode }\end{aligned}$

\section{Introduction}

The preference of small homes over larger, more expensive dwellings is a reoccurring trend which has surfaced 1 frequently in the last century and has peaked in recent years in reaction to the mortgage crisis, economic recession, increased scarcity of resources, and increased crowding in cities. ${ }^{1}$ A growing number of consumers seek to "scale back" to smaller houses which are "simple, affordable, ecologically sustainable"2 while maintaining certain levels of comfort and productivity. As a result of this burgeoning consumer market and considerable media attention, the current "small home" and "tiny house" movements have seen the development of thousands of residences well under 500 square feet and the development and implementation of novel space saving techniques and technologies between industry and the "do-it-yourself" community. Residences which have implemented these solutions are shown including small homes, cabins, apartments, micro-apartments, house boats, boats, recreational vehicles, and living quarters on board military vessels (See Ref. 1, 3, and 4 for examples).

There are several similarities between the design problems of small, compact residences and space habitats which would make novel terrestrial small space design solutions applicable to space habitat design. Both seek to: 
- support human living and working tasks in a confined space without adversely affecting their physiological, psychological, and sociological health

- $\quad$ integrate multiple tasks and accommodations into small, compact layouts where all tasks cannot have

dedicated volume and privacy may be limited

- $\quad$ minimize the amount of materials, mass, and cost

- $\quad$ enable inhabitant self-sufficiency and reuse materials for multiple purposes

- $\quad$ enable humans to survive in remote locations

- $\quad$ enable mobility (when desired)

This paper identifies several small space design techniques and technologies which can potentially improve space habitat mass, volume utilization, interior layout functionality, and habitability. These cross-cutting architectural solutions will enhance current habitat designs, enabling long duration human exploration missions. The solutions are grouped into three categories: 1) those which directly affect the space habitat interior layout design (e.g., spatial relations, multipurpose use of space, functional area adjacencies, etc.), 2) those which impact the design of furniture and hardware to be packaged within the habitat interior (e.g., reconfigurable equipment, novel stowage methods, etc.), and 3) those related to electronics and computing devices which have benefitted small space designs (home automation, mobile devices, etc.). In Section II, these solutions are described in detail including discussion of how they save interior volume or mitigate other small space issues such as confinement stress and crowding. Specific limitations or implementation details necessary for applying these techniques to space habitat designs are also discussed. Finally, in Section III, concrete recommendations are given based upon a qualitative assessment of the viability of implementing the various solutions and their potential to substantially impact future human missions.

\section{Cross-Cutting Architectural Solutions}

The intent of this paper is to describe solutions developed in the small space design community which can benefit space habitat designs, with a specific emphasis on long duration habitats being designed for interplanetary transits and planetary surfaces. This benefit can be assessed through a several established habitat design criteria including:

- $\quad$ reduced mass $5^{5,6,8}$

- $\quad$ reduced complexity

- $\quad$ increased open and accessible volume/floor areas (or habitable volume) 5, $, 7,8,9^{-1}$

- $\quad$ reduction of wasted or unusable volume $e^{5,6,9}$

- $\quad$ increased stowage and stowage access ${ }^{5,6,8,9}$

- increased surface area access $8^{8,9}$

- $\quad$ improved control over the environment ${ }^{7}$

- $\quad$ increased visible volume and lengths of lines of sight $^{5,6,7,8,9}$
- $\quad$ improved circulation (translation efficiency) $)^{5,6,7,8,9}$

- $\quad$ improved private and personal space s,6,7 $^{5}$

- multifunctional use of space and equipment ${ }^{5,6,8,9}$

- $\quad$ improved workspace utility ${ }^{5,7}$

- $\quad$ reduced perceived crowdedness $5,6,7,8$

- $\quad$ improved sensory variability ${ }^{5,7}$

- decreased social monotony ${ }^{7}$

- improved auditory and hygiene isolation ${ }^{5,6,7,9}$

- $\quad$ improved utilities efficacy ${ }^{5,6,8}$

- improved comfort ${ }^{5,7}$

Though many of these small space design solutions may be viewed as ubiquitous aspects of sound architectural practice or may be well documented in spaceflight design literature such as Out of this World: The New Field of Space Architecture $e^{10}$ or the NASA Human Integration Design Handbook (HIDH) ${ }^{5}$, all of the major solutions identified in the small space literature which address the habitat evaluation criteria will be discussed here for completeness. Figure 1 shows the types of small space design solutions which are discussed in this paper. 


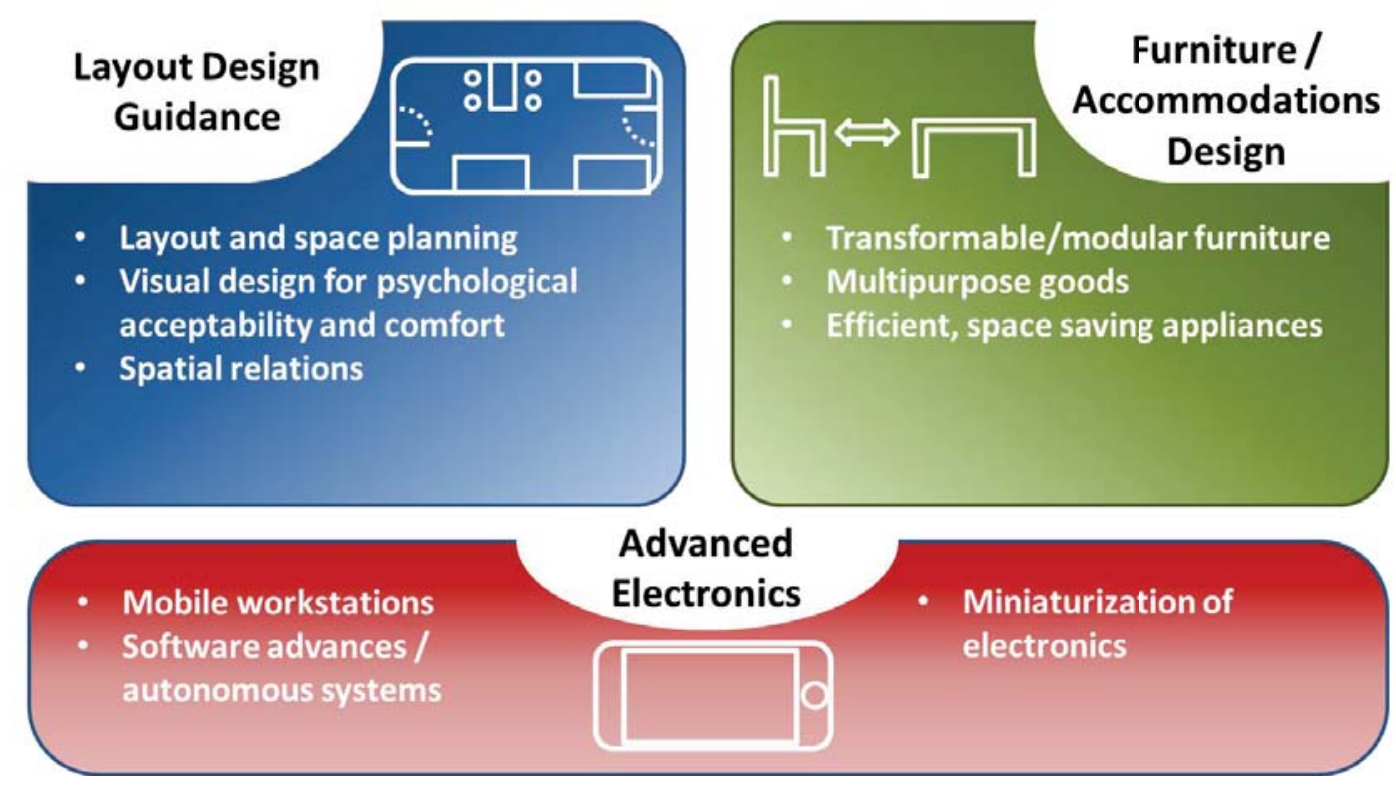

Figure 1: Categories of Small Space Design Solutions

\section{A. Layout Design Guidance}

The first category of small space solutions augments the functionality or acceptability (i.e., the feel) of a space through the design of its interior layout. The intent of layout-based solutions is to make an interior feel intentional and well designed; providing spaciousness and separation where desired, increasing the quality of private and personal space, providing good circulation to reduce crowding, and designing and arranging task areas for efficacy. ${ }^{7,11,12,13}$ Layout-based solutions fall into two basic categories: layout space planning and visual aesthetic solutions.

\section{Layout Based Solutions}

Layout space planning refers to the allocation, arrangement, and partitioning of spaces necessary for required functions, equipment, and furniture. ${ }^{7}$ At least in American culture, moderately spacious interiors with separable rooms for private, individual time are desired for comfort. ${ }^{11}$ However, in both tiny home and spacecraft design, the size of the interior is limited by the lot, trailer, launch vehicle or lander size. ${ }^{12}$ Furthermore, the small sizes of interior layouts tend to feature one contiguous space, making the accommodation of desired separate spaces difficult. In both design problems, the minimum amount of interior space required depends on the space necessary to accommodate each function, how much those functions can be overlapped or closely packed, and an assessment of how the feel of the space affects overall psychological or psychosocial health of the inhabitants. In both small space design and space habitat design, sound architectural planning and more aggressive layout solutions are required to ensure the highly integrated spaces remain livable. To some degree, the isolated confinement aspect of space habitats which prevents occupants from 'escaping' outdoors makes the selection of an adequate volume and implementation of these layout solutions even more critical.

In general architectural practice, larger spaces are provided for common areas to reinforce positive social interactions, smaller spaces are provided for private areas to establish a sense of comfort, and other spaces are rightsized according to their functions. In order to reduce the overall space required, many of the areas allocated to these functions are overlapped into multifunction spaces. One principle applied to small space design is to ensure that no space has only a single purpose and sits largely unused for any significant period of time. ${ }^{12,13}$ Often, circulation spaces serve multiple purposes or are removed from the layout entirely as in the case of bed alcoves ${ }^{11}$, which eliminate the space around the bed which serves no other purpose but circulation. A similar small space solution is to use every nook and cranny of previously unused space. In terrestrial architecture, this implies maximizing the utility of vertical space and employing built-in storage or dual function storage pieces such as chests and storage ottomans. This can be similarly adapted to space habitat design by taking full advantage of microgravity to overlap task spaces in the vertical direction as well as leveraging a changing orientation to redefine the space. For example, the vertical configuration shown in Figure 2 may be setup for a working situation, but changing the orientation and 
deploying some key visual orientation aids (furniture, lighting, etc.) can then convert the same space into a social/recreation space. This can be further augmented by visual occlusion of previous labels and orientation references to help the transition. In order to minimize wasted space in compact interiors, it is necessary to accurately plan for the amount of storage necessary while also including access and translation routes (which are common spacecraft design challenges). Another layout strategy which has been implemented to maximize the usage of space in more military applications is scheduling. While "hot bunking" has been deemed an unattractive psychological solution in spacecraft applications due to "its lack of privacy and personal space" ${ }^{14}$, consideration of the schedule and time spent in public, work, and common areas should play into the degree of multi-functionality of a space and the relative arrangement of adjacent spaces. ${ }^{5,6,7}$ Additionally, ensuring that frequently used items are readily accessible and colocated with their task areas creates a feel of intentionality that improves occupant productivity and contentment. ${ }^{7,15,16}$ Similarly, sequentially used functions, highly related functions, and functions sharing utility runs should also be colocated to simplify translations and design complexity. ${ }^{6,16}$


Figure 2: Multi-use, Multi-orientation Habitat Interior

Aside from providing adequate volume and arranging the functions to increase the utility of a space, partitioning a space into multiple, separate areas can contribute to making an interior feel spacious. ${ }^{13}$ By defining specific places for certain tasks to occur, those spaces are given an identity, and a feeling of translation between those locations becomes possible. ${ }^{11}$ As long as the locations of these spaces are carefully planned to avoid crowding, this translation in a confined space greatly contributes to the perception of layout spaciousness and utility which benefits the psychological health of occupants. ${ }^{7}$ This partitioning can be achieved by the use of room dividers, doors, walls, screens, furniture, lighting, color/finish choices, or by physically providing distance between functional spaces. Many types of separations including visual, olfactory, auditory, vibration, etc. must also be considered to provide privacy for sensitive functions such as hygiene or personal space where the individual can reestablish a sense of self. ${ }^{11}$ Even in most tiny homes where all of the functions are so colocated that partitioning the space becomes difficult, some degree of visual occlusion (partitions, walls, doors, screens, etc.) is usually provided for hygiene functions. ${ }^{12,13}$ In most living spaces, an "intimacy gradient" as shown in Figure 3 is developed where the level of privacy needed increases as one travels from the front of a house to the back. ${ }^{11}$ When achievable, this layout strategy has been demonstrated to be particularly important in habitat design and has also been extended to hygiene and noise. ${ }^{5}$

The recent small space design trend of reconfigurable interiors, which allows the occupant to repeatedly redefine the purpose of a space, is particularly applicable to space habitat design. ${ }^{7}$ In tiny home design, this is limited to conversions between work spaces, living spaces, dining spaces, and sleeping spaces by transformable or deployable furniture. ${ }^{12}$ As represented in Figure 4, small apartment designs have extended this concept to movable walls and transformable furniture (discussed further in the next section). By multi-purposing the space, a smaller overall volume becomes possible as unused items are mostly kept stowed until needed. This becomes slightly more challenging in microgravity where most systems require electronics and other utility runs, but this can be addressed by thoughtful placement of those more anchored elements to the outer walls. A space habitat concept which similarly provides compact multi-purpose spaces should be investigated further. 

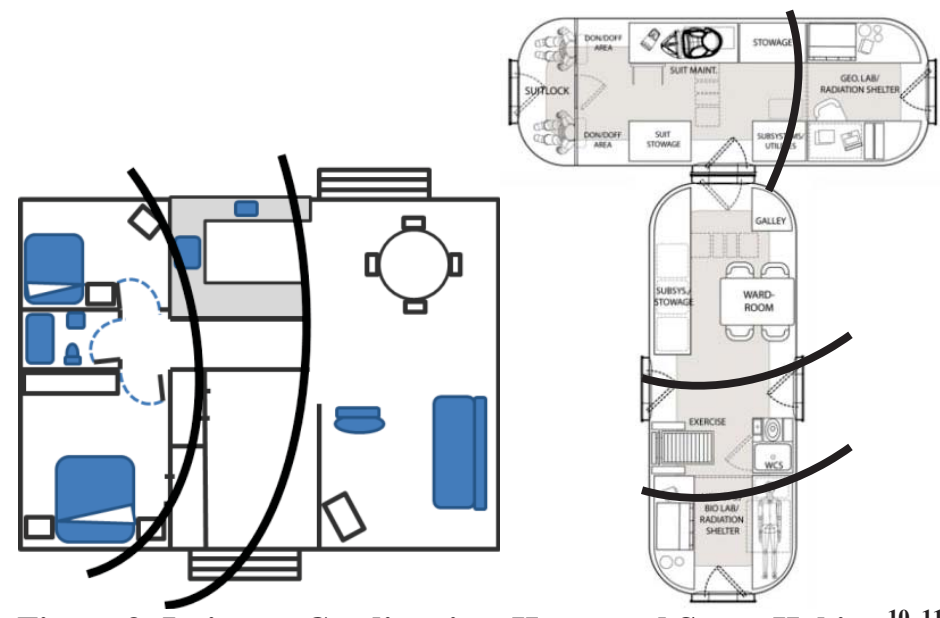

Figure 3: Intimacy Gradient in a House and Space Habitat ${ }^{10,11}$
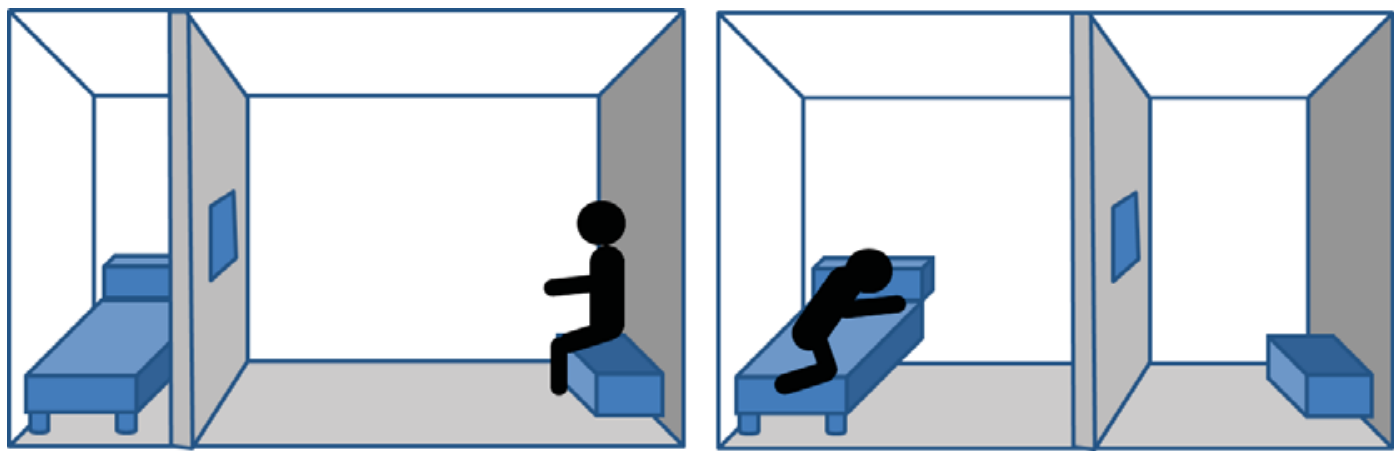

Figure 4: Movable Walls to Conserve Space (See Ref. 17 and 18 for Further Examples)

\section{Visual Aesthetic Solutions}

The visual feel of a confined space is perhaps one of the most significant habitat design takeaways from the small space design movement. Years of designing small spaces to improve the aesthetic feel of interiors has resulted in a set of formalized design guidance which can influence space habitat design:

- Clean, uncluttered space with strong visual cues and sight lines ${ }^{19,20}$ : Messes are more easily noticed in small spaces because they interrupt the sight lines and visual cues which help to define the space. By keeping a clean, uncluttered space with strong visual cues, the eye can be drawn to desired aspects of the space like large ceilings or spaciousness. This solution is closely tied to better stowable accommodations. As seen in Figure 5, this is a particular aspect of space habitat design which has yet to be put into practice since most spacecraft have several intrusions into the open volume (deployed workspaces, exercise equipment, and cables).

- Long sight lines, diagonal views ${ }^{21}$ : The diagonal view or longest sight line becomes a focus which influences the perception of room size, where longer lines of sight correspond to larger spaces. It is also a driving consideration for sensory monotony to prevent depth perception monotony. ${ }^{7}$

- Uncluttered space above waist height/half walls ${ }^{11,22}$ : Anything above waist height projects into the perceived living space, reducing the perception of openness. Also, preventing rooms (or separate areas) from being completely open or closed to one another provides a continuity of the space with the separations discussed earlier.

- $\quad$ Ratio of ceiling height to room size ${ }^{12,21}$ : High ceilings draw the eye up to underutilized space, which gives the perception of increased room volume so long as the dimensions of a room are larger than the dimensions of the ceiling. If the dimensions of the ceiling are the largest dimension, the focus of the observer is how tall the ceilings are instead of how spacious the room is.

- Use of lighter colors with dark accents ${ }^{11,19}$ : Brighter areas generally make a space feel more open and less small. By providing a dark accent wall, a contrast is created which helps to emphasize the bright areas.

- Increased natural light replicated through natural delivery mechanisms ${ }^{12,21}$ : Besides the circadian benefits from exposure to natural light that generally increase mood and restfulness, natural light gives the 
perception that a larger space is waiting outside. As natural light is limited on spacecraft, it can be replicated artificially through the use of window-like lighting devices to provide a psychological reminder of windows in terrestrial spaces.

- Use of mirrors, windows, and virtual windows ${ }^{12,22,23}$ : Mirrors can increase the perceived space by replicating an extension of a room, increasing sight lines, and bringing more light into an interior. Windows provide views of the Earth and exploration destinations while virtual windows provide sensory stimuli and perception of outside spaces.

- Providing negative space/transparent furniture: In terrestrial designs, elevating accommodations off the floor creates negative space which allows light and sight lines to reach more visible space. Similarly, designing habitats with some negative space and transparent furniture to prevent the interruption of sight lines can increase the perceived space.

- Use of natural materials/scenery ${ }^{23}$ : Several types of natural materials including natural materials like wood, stone, and plants can provide several types of sensory stimulation (visual, tactile, olfactory, gustatory, etc.). These materials and natural scenes have been proven to reduce stress associated with isolated and confined environments. ${ }^{24}$
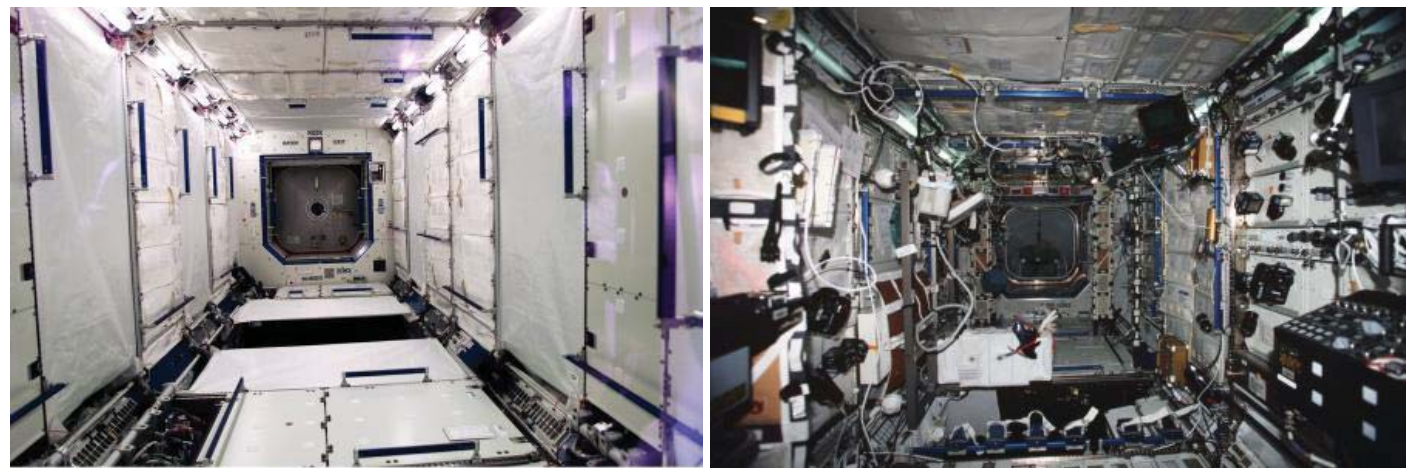

Figure 5: International Space Station US Lab Interior on Delivery and After Deployment (NASA)

\section{B. Furniture/Accommodations Design}

To facilitate the design of small space layouts which are optimized for spacious feel and functionality, it is often necessary to include specially designed furniture and or novel technologies in its construction. There are several types of furniture and accommodations which have been built in order to save space or improve the quality of small space interior designs which have applicability to the long duration space habitat problem. These include: movable walls, transformable/reconfigurable/deployable/stowable furniture, low wasted-space stowage solutions, and efficient space-saving appliances.

\section{Movable Walls}

Figure 4 shows the benefit of movable walls. These allow compact storage of functions when not in use and facilitate transformation of the space into multiple configurations for multiple operational situations. Intermittently used functions like food preparation, hygiene functions, and sleep quarters are primary candidates for this type of stowage. One desirable feature is the ability to deploy functional pieces of furniture with minimal effort, time, and disruption to other spaces. This concept has the potential to significantly impact habitat interior design by making the overall volume smaller and increasing the ability to overlap functional volumes with minimal deployment complexity. Some thought has been given to lightweight, deployable, personal sleep quarters in previous habitation work, but challenges such as ventilation and utility runs remain obstacles to creating fully transformable interior habitats. However, technological innovations developed for these transformable apartments such as track mounting movement rails with power and low force mechanisms can potentially enable the movable wall concept for space habitats in the future.

\section{Transformable/Reconfigurable/Deployable/Stowable Furniture}

The consumer market for smaller homes has created a market for furnishings which match the scale of small interiors and increase their utilities. Hundreds of companies have created novel types of furnishings which serve multiple purposes and are stowed more compactly. As shown in Figure 6, transformable pieces can substantially save space by combining multiple rooms into one. ${ }^{25}$ Many of these furniture pieces do not translate directly to the 
space environment, where microgravity eliminates the usability of most surfaces. However, the principles utilized by these pieces still apply, and the modified versions of this furniture may still be utilized for planetary surface missions. In particular, modular assembly techniques can be leveraged from furniture in manners similar to Howe's Logistics to Living Concept shown in Figure 7. Also, the concept of stowed sleeping quarters translates well to space habitat design. Unless workstations are integrated into crew quarters, this space is mostly wasted during waking hours. Methods of collapsing this space while maintaining the location of personal effects and allowing for quick deployment of this space for private communications would be able to free up anywhere from 8-15 cubic meters of internal volume based upon International Space Station crew quarters. Additional research into partition deployment and foldable structures should be pursued to enable concepts like the one shown in Figure 8.
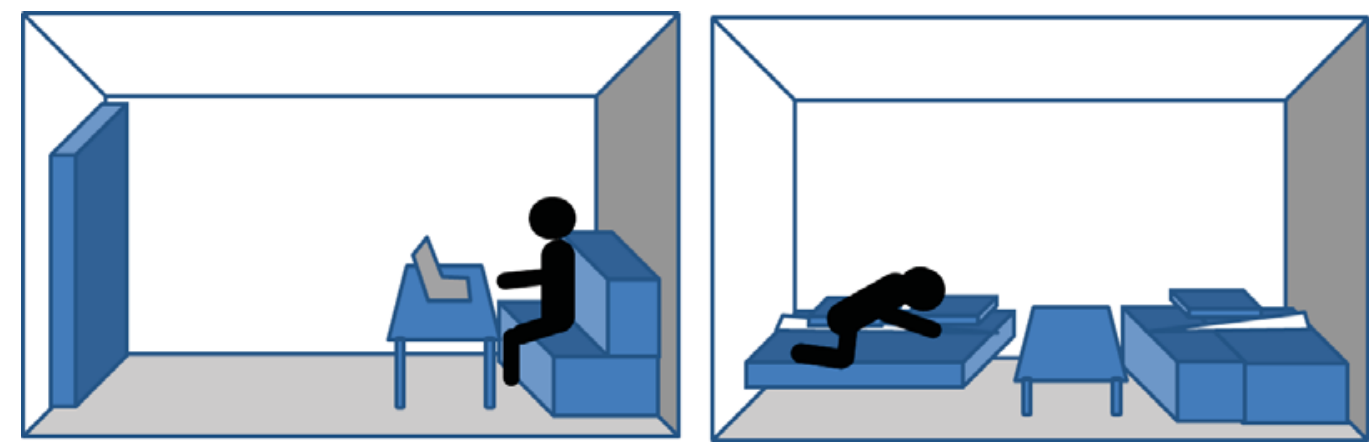

Figure 6: Transformable Furniture

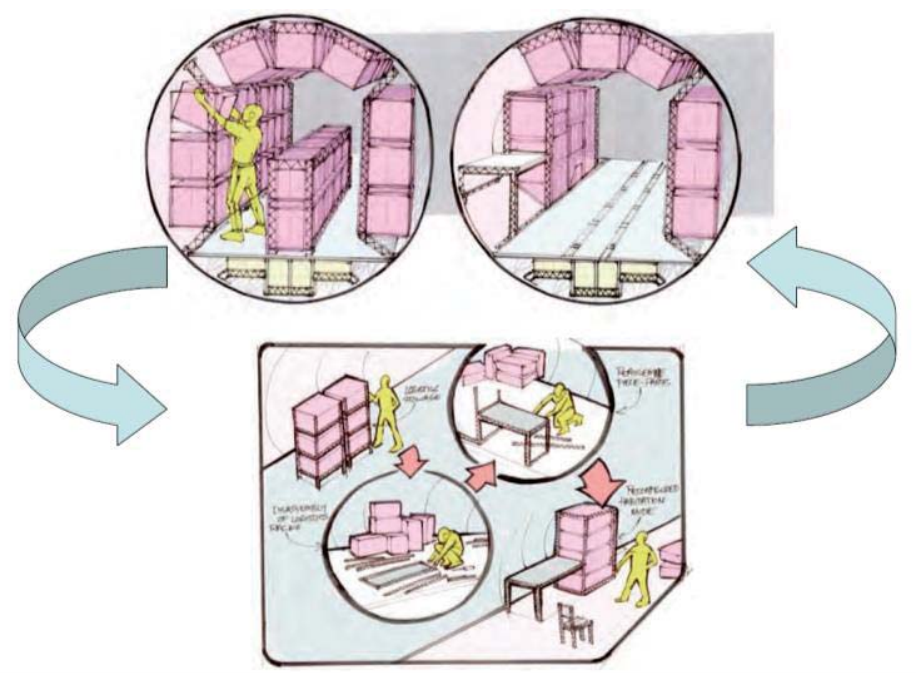

Figure 7: Logistics Packaging Repurposed as Kit-of-Parts Furniture ${ }^{26}$
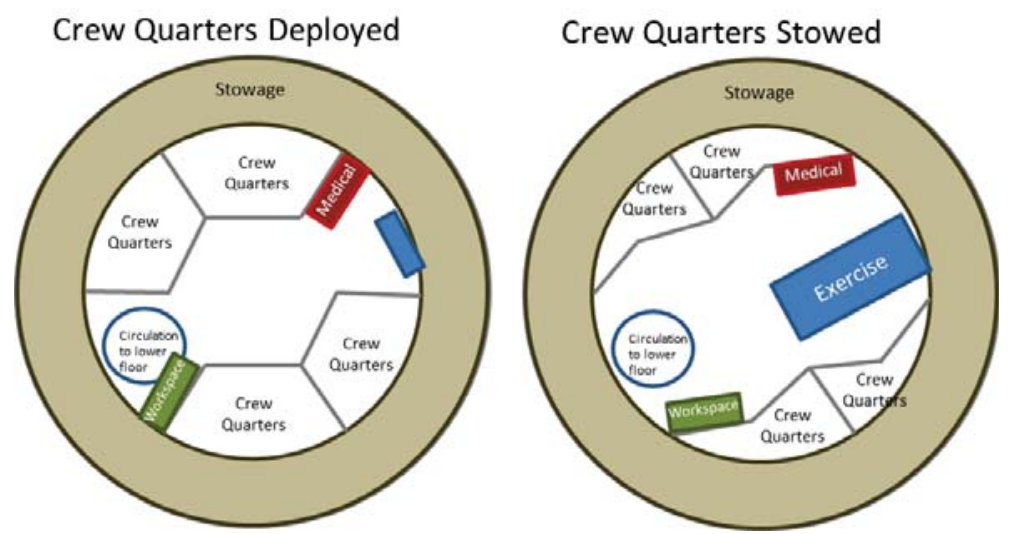

Figure 8: Internal Layout Demonstrating the Potential Benefit of Transformable Crew Quarters with Stowable Exercise Equipment 


\section{Low Wasted-Space Stowage Solutions}

One of the most cited techniques to make a small space feel larger is better storage of goods and equipment. Small space design storage can reduce the dedicated volume necessary for stowage, which can reduce the overall footprint of a space and remove/hide clutter which improves the perception of spaciousness ${ }^{12}{ }^{27}$. This is especially important in space habitat design where all of the goods necessary for the duration of the mission (typically months to years) must be compactly stowed and readily accessible within the vehicle. In addition, planetary protection measures often require the habitat to store all used items safely like trash and expended spares, which requires some intermediary stowage prior to long term stowage. This can also create unique challenges when locations previously allocated for storage of fresh items must now store dirty or expended items without contaminating unused goods. Space habitat design requires several types of storage used in small space design. First, organized storage must be provided for long duration storage of the majority of goods such that any part or item can be found quickly with minimal crew time. Second, highly flexible, temporary storage is necessary for the short-term goods such as food and tools which will be needed readily at hand. Finally, uniquely designed storage is necessary to take advantage of previously wasted spaces like endcaps without imposing large access requirements (similar to rotating shelves in corner cabinets in kitchens). Novel design solutions leveraging small space furniture and existing spacecraft storage solutions should be combined into highly flexible spacecraft storage solutions like the Random Access Framework concept developed by the Jet Propulsion Laboratory shown in Figure 9.

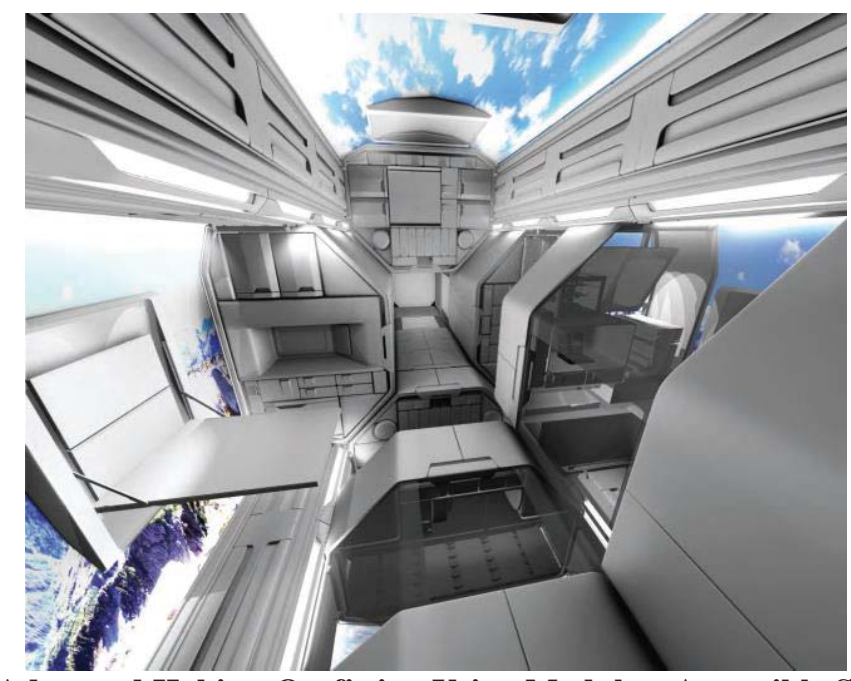

Figure 9: Advanced Habitat Outfitting Using Modular, Accessible Storage ${ }^{28}$

\section{Efficient, Space Saving Appliances}

Another advance in small space design is the development of efficient small space appliances which allow the development of smaller kitchens and bathrooms. These compact appliances are potentially applicable to long duration planetary exploration efforts where the gravity can potentially enable their operation similar to Earth. Even though some of these technologies may not be directly usable for microgravity missions due to the unique challenges of operating in microgravity, the technologies and software developed for these appliances may still provide useful insight to facilitate the design of new, in-space equivalent appliances.

\section{Advanced Electronics}

Electronics and computing have advanced significantly in the past couple decades. Laptops today are as capable as supercomputers in the 80 s, and mobile devices drive a significant portion of the consumer market. Mobile devices has developed increased battery life, increased computing power, increased data storage, and the ability to wirelessly transmit data and interact with household electronics. The combination of the internet and cell phone service has made a vast amount of information on every topic readily accessible to the public, including a large amount of "doit-yourself" information on every aspect of home design and construction. Digital media and slim LED televisions have drastically reduced the volume necessary for entertainment in the home. Though these and many more advances were not spawned by the small homes movement, they've had a significant impact on the design and accessibility of those spaces. Mobile devices and laptops have replaced televisions and large media libraries in many of these spaces. Wireless hotspots over cellular signals have enabled remote location and minimal wired utilities. 
Most tiny homes have been developed to run off of commercially available solar arrays, which are continually improving in performance, robustness, and cost to meet the consumer market. High capacity batteries provide backup power when sunlight is unavailable.

Mobile computing and autonomous home control systems represent the two main electronic devices adopted by small space designers which are directly applicable to improved space habitat design. First, mobile devices and laptop computing power represent a miniaturization of standard electronics which can drastically reduce the volume necessary for many systems on a habitat including environment and life support control, navigation, spacecraft control, vehicle health monitoring systems and sensors, communications equipment, entertainment, medical records systems, and scheduling/training platforms. Many of these functions can be operated from a laptop and can be wirelessly monitored and controlled from small mobile devices. Secondly, autonomous home control systems have a substantial applicability to the design of integrated vehicle health monitoring and control systems. For example, Belkin's WeMo ${ }^{29}$ home automation products enable home owners to monitor electrical usage, adjust lights, access live video data, and control/schedule almost any electrical device in their home. Similar software and devices can be used to control the spacecraft and receive real time performance of its systems to address necessary repairs. Additionally, the combination of cameras and mobile devices with accelerometers and physiological monitoring hardware can track astronaut performance and health to intelligently notify crew of their performance or potential issues resulting from spaceflight. Additional devices with potential applicability to habitat design include the ultrathin LED screens for virtual windows and surface image variability, new LED lighting technologies, and commercial solar arrays.

\section{Conclusions}

Based upon the review of small space design solutions above, several space habitat design solutions can be recommended to improve habitability, mass, volume, and cost:

- The design aesthetics of small space design should be more strongly reflected in space habitat design. These represent proven strategies based upon terrestrial space analogs to improve contentment and comfort within confined environments. This requires early design consideration of spacecraft interiors to prevent a repetition of the ISS design example from Figure 5. At a minimum, space habitat designs should definitely implement low mass solutions such as utilizing light colors, natural light sources, and natural materials to improve perception of the space and implement virtual windows to replace the "perception of outside space" which has been so critical to small space designs.

- $\quad$ Put substantial effort into design of appropriate stowage solutions (such as general stowage for transitioning items and hidden stowage) to ensure that the visual aesthetic of interior spaces is not compromised during spacecraft lifetime. This requires an emphasis on psychological acceptability of the space and the implementation of low mass design solutions.

- Reconfigurable interiors represent potentially substantial savings of internal volume, but also require detailed scheduling and layout analyses. Specific solutions which should be investigated in more detail with sample designs include movable walls similar to Figure 4 and furniture construction techniques from Ref. 26.

- Commercial-off-the-shelf hardware and software for autonomous control of homes should be assessed for applicability to space habitats. Anticipated developments in the coming years in this field could negate the need for specialized in house software.

Other future work tasks should be applied to better prioritize the application of small space design solutions to space habitats. First, integrated conceptual design tools which can assess the effectiveness of these solutions in an integrated habitat design should be developed. These include mass sizing and layout analysis models similar to those proposed in Ref. 6. Second, several small space layouts should be assessed with these tools to derive formalized guidance on layout measurements. For example: several small space layouts can be investigated to derive statistics on the most compatible functional areas to overlap such as dining/ living room/office or bedroom/living room.

\section{References}

${ }^{1}$ Kahn, L., Tiny Homes: Simple Shelter: Scaling Back in the $21^{\text {st }}$ Century, Shelter Publications, Bolinas, California, 2012, pp. vivii.

${ }^{2}$ Rowan, G., Compact Houses, 50 Creative Floor Plans for Well-Designed Small Homes, Storey Publishing, October 8th 2013, pp 6-7. 
${ }^{3}$ Apartment Therapy, "Living Large in an Airstream Trailer", http://www.apartmenttherapy.com/matthews-refurbished-retro-air140718, Accessed June 24, 2014 (image).

${ }^{4}$ Submarine Images from Google Street View of HMS Ocelot, Chatham, United Kingdom, https://maps.google.co.uk/maps?ll=51.395495,0.5267050000000001\&cbp=,256.06,0,0.0 \&layer $=$ c\&panoid $=$ D03HgqkhWFIAAAQJOMnFiA\&spn $=0.18000000000000788,0.3000000000000001 \&$ output $=$ classic \&cbll=51.395495,0.526705\&dg=ntvb, Accessed June 25, 2014.

${ }^{5}$ NASA, "Human Integration Design Handbook", NASA/SP-2010-3407, 2010.

${ }^{6}$ Simon, M., Wilhite, A., "A Tool for Automated Design and Evaluation of Habitat Interior Layouts", AIAA SPACE 2014 Conference and Exposition, San Diego CA, 2013.

${ }^{7}$ Simon, M., Whitmire, A., Otto, C., Ed.: Neubek, D., "Factors Impacting Habitable Volume Requirements: Results from the 2011 Habitable Volume Workshop", NASA/TM-2011-217352, NASA, Houston, April 11-21, 2011.

${ }^{8}$ SICSA, "Report 1: Figure of Merit Criteria for Evaluating and Selecting Lunar Habitat module Concepts", SICSA Report, University of Houston, Houston, TX, 2008.

${ }^{9}$ Adams, C., McCurdy, M., "Habitability in Advanced Space Mission Design, Part Two: Evaluation of Habitation Elements." In S. Johnson, K. Chua, R. Galloway, P. Richter (Eds.), Space 2000 (p. 72-88). 7th International Conference and Exposition on Engineering, Construction, Operations, and Business in Space, Albuquerque, New Mexico, USA, 27 February - 2 March 2000.

${ }^{10}$ Howe, A. S. and Sherwood, B., Out of this World: A New Field of Space Architecture, AIAA, 2009.

${ }^{11}$ Alexander, C., Ishikawa, S., Silverstein, M., A Pattern Language: Towns, Buildings, Construction, Oxford University Press, United States of America, 1977.

${ }^{12}$ Mitchell, R., Tiny House Living: Ideas for Building and Living Well in Less Than 400 Square Feet,

${ }^{13}$ Gillingham-Ryan, M., Laban, J., Slater, J., Apartment Therapy Presents: Real Homes, Real People, Hundreds of Real Design Solutions, Chronicle Books, San Francisco, CA, March 5th 2008.

${ }^{14}$ Stuster, J.W. (1996). Bold Endeavors: Lessons from Polar and Space Exploration. Naval Institute Press, Annapolis, MD.

${ }^{15}$ Fitts, D., "International Space Station (ISS) Internal Volume Configuration (IVC)", AIAA 2002-6114, 1st Space Architecture Symposium (SAS 2002), Houston, Texas, USA, 10-11 October 2002.

${ }^{16}$ Simon, M., Bobskill, M.R, Wilhite, A. W. (2010). "A Structured Method for Calculating Habitable Volume for In-Space and Surface Habitats” IAC-10.A5.1.2, 61st International Astronautical Congress, Prague, CZ.

${ }^{17}$ Hong Kong Apartment Images from New York Times, http://www.nytimes.com/slideshow/2009/01/15/garden/20090115_HONGKONG_index.html?_r=0, Accessed June 25, 2014.

${ }^{18}$ Soho Apartment Images from Corcoran Group Real Estate Property listing, http://www.corcoran.com/nyc/Listings/Display/3168153, Accessed June 25, 2014.

${ }^{19}$ Ching, F., Architecture: Form, Space, and Order, John Wiley, United States of America, 2007.

${ }^{20}$ Mihai-Cristian, M., “5 tips for Fooling the Eye and Making a Room Look Bigger”, http://freshome.com/2007/05/29/5-tips-forfooling-the-eye-and-making-a-room-look-bigger/, Accessed June 25, 2014.

${ }^{21}$ Susanka, S., The not so big house: A Blueprint for the Way We Really Live, The Taunton Press, Inc., 2008

22 "Openness: 7 Spacious Tiny House Design Tips", http://tinyhousetalk.com/7-tiny-house-design-tips/ , Accessed June 25, 2014.

${ }^{23}$ Bachman, K., Otto, C., Leveton, L., "Countermeasures to Mitigate the Negative Impact of Sensory Deprivation and Social Isolation in Long-Duration Space Flight”, NASA/TM-2012-217365, NASA, 2012.

${ }^{24}$ Blake, D., "The Construction of Halley VI Station in Antarctica" (oral presentation), 10th Annual Polar Technology Conference, Bloomington, Indiana, 2014.

${ }^{25}$ Transformable Furniture Images from Resource Furniture, http://resourcefurniture.com/, Accessed June 26, 2014.

${ }^{26}$ Howe, A.S., Howard, R., "Dual Use of Packaging on the Moon: Logistics-2-Living", AIAA 2010-6049 40 th International Conference on Environmental Systems, AIAA, Barcelona, Spain, 2010.

${ }^{27}$ Green, P., "Selling the Pared-Down Life: The Founder of TreeHugger and His Apartment of the Future", New York Times, http://www.nytimes.com/slideshow/2012/05/17/garden/20120517-HOUSEOFFUTURE-6.html, Accessed June 26, 2014.

${ }^{28}$ Howe, A.S., Polit-Casillas, R., "Random Access Frames (RAF): Alternative to Rack and Standoff for Deep Space Habitat Outfitting”, ICES-2014-038, 44 ${ }^{\text {th }}$ International Conference on Environmental Systems, Tuscon, Arizona, 13-17 July, 2014.

${ }^{29}$ Belkin Home Automation: WeMo Home Automation System, http://www.belkin.com/us/Products/home-automation/c/wemohome-automation/, accessed June 26, 2014. 